A thermal sensor and switch based on a plasma polymer/ZnO suspended nanobelt bimorph structure

This article has been downloaded from IOPscience. Please scroll down to see the full text article.

2009 Nanotechnology 20065502

(http://iopscience.iop.org/0957-4484/20/6/065502)

The Table of Contents and more related content is available

Download details:

IP Address: 140.112.113.225

The article was downloaded on 06/02/2009 at 04:32

Please note that terms and conditions apply. 


\title{
A thermal sensor and switch based on a plasma polymer/ZnO suspended nanobelt bimorph structure
}

\author{
Jr-Hau He ${ }^{1}$, Srikanth Singamaneni ${ }^{2}$, Chih H Ho ${ }^{1}$, Yen-Hsi Lin ${ }^{2}$, \\ Michael E McConney ${ }^{2}$ and Vladimir V Tsukruk ${ }^{2}$ \\ ${ }^{1}$ Institute of Photonics and Optoelectronics, and Department of Electrical Engineering, \\ National Taiwan University, Taipei, 106, Taiwan \\ ${ }^{2}$ School of Materials Science and Engineering, Georgia Institute of Technology, Atlanta, \\ GA 30332-0245, USA \\ E-mail: jhhe@cc.ee.ntu.edu.tw
}

Received 31 July 2008, in final form 3 December 2008

Published 15 January 2009

Online at stacks.iop.org/Nano/20/065502

\begin{abstract}
The combination of design and subsequent fabrication of organic/inorganic nanostructures creates an effective way to combine the favorable traits of both to achieve a desired device performance. We demonstrate a miniature electrical read-out, and a sensitive temperature sensor/switch, based on a $\mathrm{ZnO}$ nanobelt/plasma-polymerized benzonitrile bimorph structure. A new read-out technique based on the change in the electric current flowing through the bimorph and the contact pad has been employed, replacing the conventional cumbersome piezoresistive method or tedious optical alignment. The thermal sensor demonstrated here has great prospects for thermal switching and triggered detection owing to the relative ease in the fabrication of arrays and the direct electrical read-out.
\end{abstract}

(Some figures in this article are in colour only in the electronic version)

\section{Introduction}

Recently there has been an explosive interest in the fabrication and assembly of novel nanostructures with welldefined sizes, shapes, and composition, which determine the properties of these nanostructures and their final applications. The current challenge is to devise methods to effectively assemble the nanostructures into functional systems and subsystems. The unique combination of piezoelectric and semiconducting properties makes $\mathrm{ZnO}$ nanostructures one of the most prospective nanomaterials [1-8]. Fine control over the growth of complex $\mathrm{ZnO}$ nanostructures such as belts, helices, rings, and flowers has been achieved by chemical vapor deposition and more recently by wet chemical methods [1, 2]. Significant progress has been made in the application of $\mathrm{ZnO}$ nanostructures [3] in the fabrication of various electronic, optoelectronic, and sensor devices, which include nanolasers [4], photodiodes [5, 6], acoustic resonators [7], piezoelectric gated diodes [8], gas sensors [9], and nanogenerators [10]. In addition, the combination of design and subsequent fabrication of organic/inorganic nanostructures has been employed for various applications, such as nanogenerators [11], light-emitting diodes [12-14], solar cells $[15,16]$, optoelectronic switches and memory devices [17].

A thermal sensor/switch is designed to control heat transfer by making and breaking thermal contact between two thermally isolated areas. Miniature, highly sensitive temperature sensors are extremely important in various civilian, industrial, and defense applications. For instance, a temperature sensor combined with actuation capability which can make and break thermal contacts at a desired temperature is highly desirable in automated systems, alarms, and process control units. A nanothermometer has recently been demonstrated in which carbon nanotubes have been filled with liquid Ga substituting the mercury in conventional thermometer [18, 19]. Microcantilever sensors, microscopic analogs of traditional bimetallic structures, have been employed for uncooled infrared (IR) detection [20-24]. The design uses the well-known phenomenon of temperatureinduced bending (due to thermally induced stress) of bimorph 
beams composed of two materials with different thermal expansion coefficients. For over 200 years, the coiled bimetallic strip thermometer has been used with little change and it is still useful today as common household thermostats and cooking thermometers.

There is a renewed interest in bimorph structures due to the advent of microcantilever systems. These systems have found a prominent place in various sensing applications due to several attractive features, namely, high sensitivity, miniature size, low cost, low power consumption, and versatility. There have been extensive efforts to maximize the bimaterial effect, involving the optimization of the differential thermal expansion, thermal conductivity, and mechanical properties of the cantilever and the sensitive layer. While microcantilevers have primarily been fabricated using conventional silicon microfabrication routines, various sensitive layers have been extensively investigated, including metals, homopolymer layers, and polymer composites. Very recently, we have introduced plasma polymers as the actuation layer, enabling a temperature resolution as low as $0.2 \mathrm{mK}$ [25]. Various read-out techniques such as laser-beam deflection, interferometry, piezoresistive, piezoelectric, capacitance, and field-effect transistors have been exploited to detect the bimorph deflections [25-29]. All of the above-mentioned detection methods involve either complex microfabrication steps or tedious optical alignment.

In the present work, we demonstrate an electrical readout temperature sensor which also acts as a thermal switch based on the bimorph structure of a $\mathrm{ZnO}$ nanobelt (NB) and plasma-polymerized benzonitrile (PP-BN). The working principle of the thermal sensor demonstrated here is based on the differential thermal expansion of the $\mathrm{ZnO} \mathrm{NB}$ and the plasma polymer layer. The change of electrical current of the bimorph is initiated by the deflection of the bimorph at a particular critical temperature, which results in the change of the contact area of the $\mathrm{ZnO} \mathrm{NB}$ and the Au metal pad (contact metal). This bimorph structure can also act as a switch at a predetermined temperature through an appropriate choice of the thickness of the $\mathrm{ZnO} \mathrm{NB}$ and the polymer layer.

\section{Experimental details}

Details of the vapor-liquid-solid growth technique employed for the fabrication of the $\mathrm{ZnO} \mathrm{NBs}$ has been reported elsewhere [30]. The synthesized $\mathrm{ZnO} \mathrm{NBs}$ are transferred from the $\mathrm{Al}_{2} \mathrm{O}_{3}$ substrate to prepatterned $\mathrm{Ti} / \mathrm{Au}$ electrodes by touching the NB sample with the electrodes. A single $\mathrm{ZnO} \mathrm{NB}$ device lying across two electrodes could be easily achieved using this method. To reduce the contact resistance and customize the length of the $\mathrm{ZnO} \mathrm{NB}$ device, a focused ion beam is employed to deposit a conducting mixture of Pt$\mathrm{Ga}$ on the contacts between the $\mathrm{ZnO}$ and $\mathrm{Ti} / \mathrm{Au}$ electrodes. $\mathrm{Ti} / \mathrm{Au}$ electrodes for current-voltage $(I-V)$ measurements were fabricated on $100 \mathrm{~nm} \mathrm{Si}_{3} \mathrm{~N}_{4} / \mathrm{Si}$ wafer through a typical process described in the literature [31]. The electrode pattern was designed to have a few parallel electrodes separated by 5-20 $\mu \mathrm{m}$. The oxygen plasma treatment was carried out to eliminate the surface contamination of the $\mathrm{ZnO} \mathrm{NB}$ device

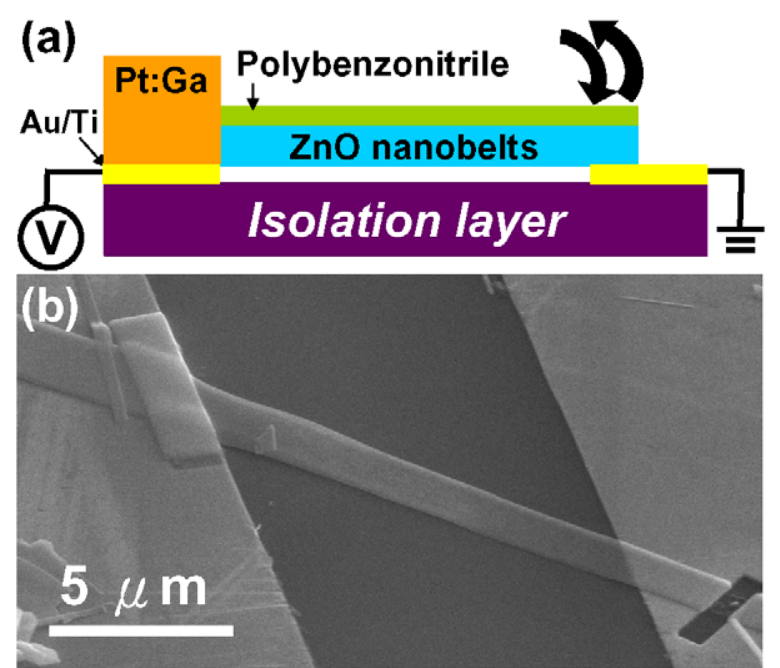

(c)

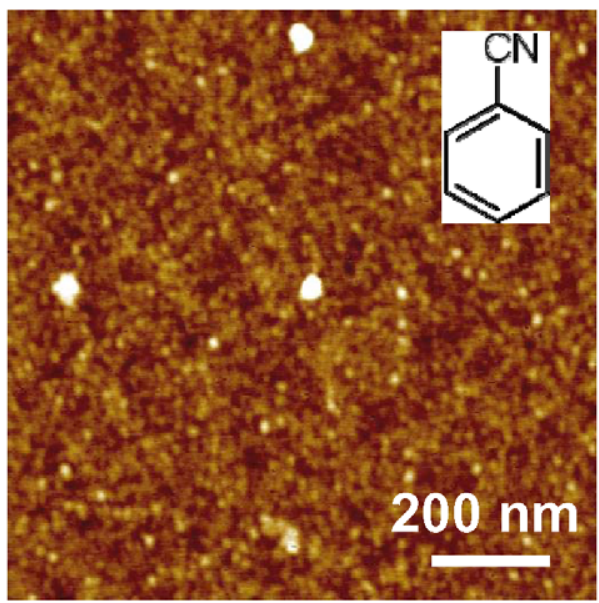

Figure 1. (a) Schematic representation of the polymer coated $\mathrm{ZnO}$ nanobelt as a thermal microswitch device. (b) A representative SEM image of $\mathrm{ZnO}$ nanobelt device. (c) AFM image showing the morphology of the plasma-polymerized benzonitrile. The $Z$ range is $5 \mathrm{~nm}$. The inset shows the chemical structure of PP-BN.

before depositing PP-BN (the chemical structure is shown as the inset of figure $1(\mathrm{c})$ ). The NB device was coated with PP$\mathrm{BN}$ using plasma enhanced chemical vapor deposition with argon (50-200 $\left.\mathrm{cm}^{3} \mathrm{~min}^{-1}, 99.999 \%\right)$ as the carrier gas within a custom-built chamber as described in detail elsewhere [32]. The thickness of the plasma polymer coating was measured with an atomic force microscope (AFM) by scanning at the edge of the film. The AFM surface morphology and thickness measurement were performed using a DI-3000 AFM in tapping mode [33].

Theoretical values for the deflections of the NB/PP-BN bimorph structure have been estimated using finite element analysis (FEA) executed in the structural mechanics module of a commercial package (COMSOL Multiphysics 3.2). A typical FEA involves the reduction of the energy functional $(E)$ of individual elements of the model. The minimization is attained by setting the derivative of $E$ with respect to the displacement of the mesh element to zero. The principle of virtual work was selected as the implementation method for minimizing $E$. The model used a nonlinear mode to account for the probable saturation effects in the deformation. 

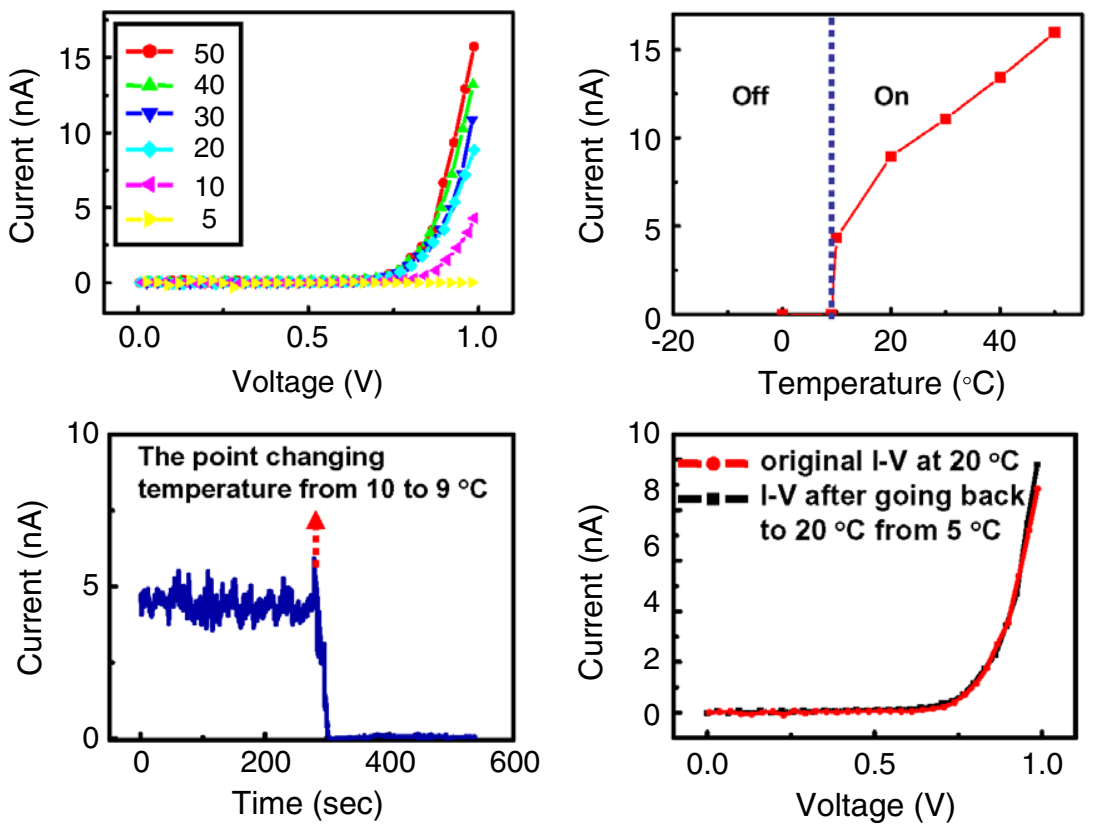

Figure 2. (a) Temperature-dependent $I-V$ measurements. (b) Current-temperature curve recorded at the forward bias of 1 V in agreement with a temperature microswitch. (c) Current-time measurement. The arrow shows the starting point for changing the temperature from 10 to $9^{\circ} \mathrm{C}$. (d) The original $I-V$ measurement at $20^{\circ} \mathrm{C}$ and $I-V$ measurement after heating to $20^{\circ} \mathrm{C}$ from $5^{\circ} \mathrm{C}$.

\section{Results and discussion}

Figure 1(a) shows the schematic representation of the nanosensor/nanoswitch device, in which a $\mathrm{ZnO} \mathrm{NB}$ bridges two electrodes with one of the ends clamped to the electrode underneath. The device architecture is similar to a conventional bimaterial microcantilever structure except that the free end of the $\mathrm{ZnO} \mathrm{NB}$ rests on the electrode. Figure 1(b) shows the scanning electron microscope (SEM) image of the NB suspended across the two contact pads. The constrained and free end of the $\mathrm{ZnO} \mathrm{NB}$ is identified on the SEM image. AFM imaging was performed directly on the $\mathrm{ZnO} \mathrm{NB}$ to study the uniformity of the coating and surface morphology of the plasma polymer film. Figure 1(c) shows the AFM surface morphology of the PP-BN, depicting a uniform coating and the characteristic grainy surface morphology and low microroughness (below $2 \mathrm{~nm}$ within a $1 \mu \mathrm{m}^{2}$ area).

While the typical $\mathrm{ZnO}-\mathrm{Au}$ contact is Schottky in nature, depositing a Pt-Ga mixture between the NB and Au electrodes usually results in ohmic characteristics [34]. The mixture provides low contact resistance of $\mathrm{Pt}-\mathrm{Ga} / \mathrm{ZnO}$ compared to the ultra-high contact resistance of pure $\mathrm{Pt} / \mathrm{ZnO}$ reported in the literature [35]. The $I-V$ characteristics of the bimorph device exhibited a temperature-dependent rectifying behavior, as shown in figure 2(a). The magnitude of the forward current at a given voltage decreases significantly with decreasing temperature.

As is known, the current through a Schottky barrier diode at a forward bias, based on thermionic emission-diffusion theory, is given by the relation [36]

$$
I=A_{\mathrm{d}} A^{* *} T^{2}\left[\mathrm{e}^{(q V / k T)}-1\right] \mathrm{e}^{\left(q \varphi_{\mathrm{b}} / k T\right)}
$$

where $A_{\mathrm{d}}$ is the diode area (the contact area of $\mathrm{ZnO} \mathrm{NB}$ and Au metal), $A^{* *}$ is the effective Richardson constant, $T$ is the temperature in Kelvin, $k$ is the Boltzmann constant, $q$ is the electronic charge, and $\varphi_{\mathrm{b}}$ is the barrier height. According to equation (1), there are two factors leading to temperaturedependent current variation. First, increasing/decreasing the temperature will lead to proportional deflection of the bimorph due to differential thermal expansion [28]. Correspondingly, the deflection of the bimorph should reduce the contact area of the $\mathrm{ZnO} \mathrm{NB}$ and $\mathrm{Au}$ metal pair, leading to an increase in the contact resistance. Second, the current is influenced directly by the $T^{2}\left[\mathrm{e}^{(q V / k T)}-1\right] \mathrm{e}^{\left(q \varphi_{\mathrm{b}} / k T\right)}$ term, which is partly attributed to the tunneling through a barrier from occupied levels, whose population is slightly modified by the elevated temperature, and a potential barrier that allows increasing penetration with increasing forward voltage. Owing to the two above-mentioned contributions, temperature-dependent $I-V$ characteristics were observed. However, as the temperature reached $5^{\circ} \mathrm{C}$, the current dramatically dropped to noise level, indicating an open circuit.

Figure 2(b) shows the current versus temperature curve at the forward bias of $1 \mathrm{~V}$, in the OFF and ON states, with the ON state clearly exhibiting a linear increase in the current with temperature. For further analysis of the thermal switch performance, the current-time measurement from 10 to $9{ }^{\circ} \mathrm{C}$ was investigated, as shown in figure $2(\mathrm{c})$. As the temperature was decreased from 10 to $9^{\circ} \mathrm{C}$, a notable sharp drop in the current was observed, corresponding to the separation of the $\mathrm{ZnO} \mathrm{NB}$ and the Au metal pad pair, resulting in $A_{\mathrm{d}}=0$. The sudden snap-off of the $\mathrm{ZnO}$ from the metal electrode as the temperature is varied within a narrow window from 10 to $9^{\circ} \mathrm{C}$ manifests itself as a thermal trigger switch.

Finally, the reversibility of the thermal bimorph nanoswitch was assessed by repeatedly cycling the switch. For testing the repeatability of this bimorph structure, the current was 


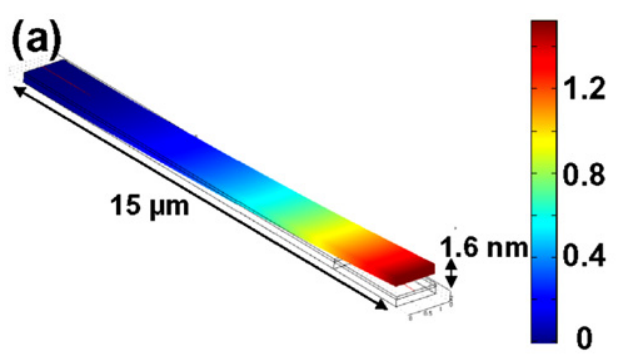

(c)

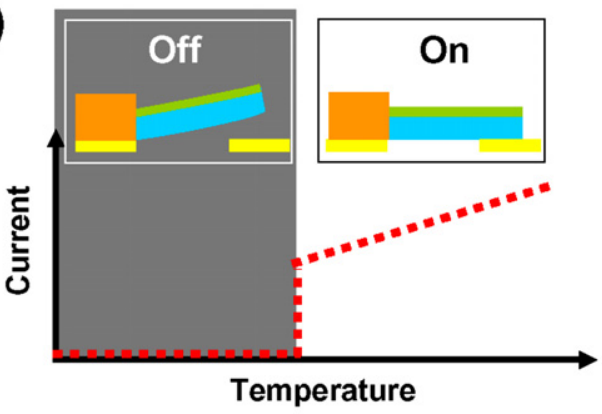

(b)

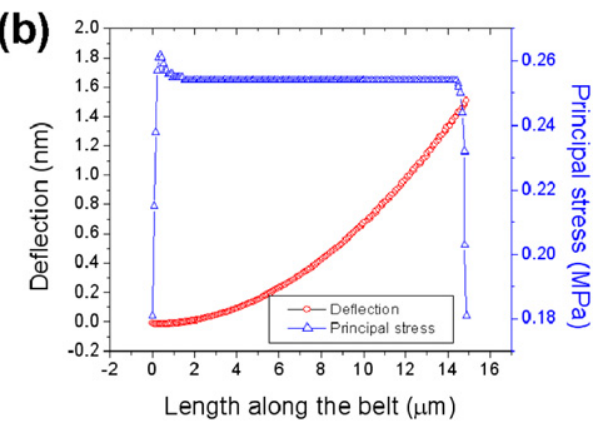

(d)

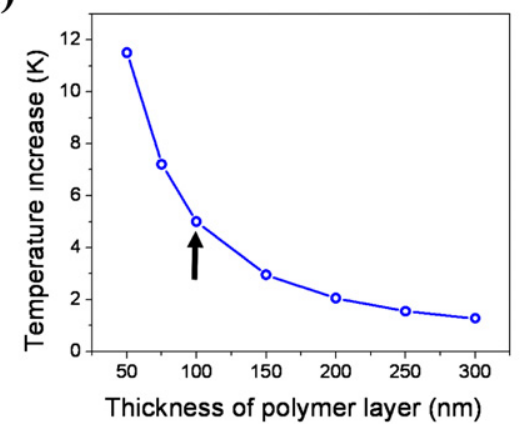

Figure 3. Finite element analysis (FEA) of the bimorph structure. (a) Deflection of the nanobelt bimorph structure for $1 \mathrm{~K}$ change in temperature assuming a free end of the cantilever. (b) Plot depicting the deflection profile along the length of the nanobelt and the principal stress at the top surface for $1 \mathrm{~K}$ change in temperature. (c) Schematic of the working principle of a thermal switch. Expected temperature dependence of the current, $I(T)$. At the moment when the metal pad and the bimorph are separated, $I(T)$ shows a switching action: a sharp drop occurs, corresponding to the current sharply reducing to 0 . (d) FEA showing the increase in temperature required to induce a deflection of $10 \mathrm{~nm}$ for various thicknesses of polymer layer on the $\mathrm{ZnO}$ nanobelt. The arrow points to the thickness of the polymer layer fabricated in this study.

measured after the temperature was changed from $5^{\circ} \mathrm{C}$ back to $20^{\circ} \mathrm{C}$. Figure $2(\mathrm{~d})$ is the comparison of $I-V$ measurement, indicating that there is no noticeable change, which demonstrates that this bimorph device can serve as an efficient thermal switch with repeatable ON/OFF cycling. Compared to time-consuming, delicate, and rather expensive observations using a transmission electron microscope (TEM) and laser-beam deflection methods [18, 25, 26], this standalone $\mathrm{ZnO} \mathrm{NB} / \mathrm{PP}-\mathrm{BN}$ bimorph device is more straightforward and can be easily tuned to different threshold levels by utilizing different thicknesses of the PP-BN coating with high thermal expansion. The interfacial thermal stresses developed within these structures allows for the thermally induced actuation caused by reversible bending deformation of a bimorph due to a vast mismatch in film properties and thermal expansion coefficients of the materials within a wide range of temperatures.

The significant difference between the NB/PP-BN bimorph structure and a conventional bimaterial cantilever structure is that in the former the free end of the NB rests on the substrate (electrode) as opposed to the freely suspended state in the latter. The differential surface stress and thus the deflection of the conventional bimaterial cantilever structure can be modeled by the modified Stoney equation [28]. However, in the case of the NB bimorph structure, interaction of the free end with the electrode plays a significant role in the deflection behavior of the NB owing to the nanoscale dimensions.

One of the primary contributions responsible for the adhesion between the $\mathrm{ZnO} \mathrm{NB}$ and the Au (electrode) is the capillary force between the two flat surfaces since the thermal switch was operated at ambient humidity conditions $(\sim 60 \%)$. The magnitude of the capillary force can be estimated from the Laplace pressure drawing the two plates together, given by $P=2 \gamma_{\mathrm{L}} / x$, where $x$ is the separation between the plates and $\gamma_{\mathrm{L}}$ is the surface tension of the water bridging the parallel plates [37]. For given geometrical parameters and a separation of $1 \mathrm{~nm}$, the Laplace pressure drawing the $\mathrm{ZnO}$ belt to the $\mathrm{Au}$ electrode is calculated to be $130 \mathrm{MPa}$ (assuming complete wetting of the Au pad and the $\mathrm{ZnO}$ belt). To compare this value with the differential stresses developed, finite element analysis (FEA) was employed to estimate the stress in the bimorph structure for the change in temperature [38]. The material properties of the $\mathrm{ZnO}$ (Young's modulus $(E)=210 \mathrm{GPa}$ and thermal expansion coefficient $(\alpha)=12 \times 10^{-6} \mathrm{~K}^{-1}$ ) have been chosen from the standard COMSOL library. The elastic modulus of the plasma polymer film was measured to be $2 \mathrm{GPa}$ by AFM nanomechanical probing [39]. The thermal expansion coefficient of the PP-AN was measured to be $100 \times 10^{-6} \mathrm{~K}^{-1}$ by measuring the thickness of the film deposited on silicon using ellipsometry between 25 and $90^{\circ} \mathrm{C}$ [38]. Figures 3(a) and (b) show the deflection profile and the surface stress on the polymer side of the bimorph structure. The bimorph structure exhibits a deflection of $\sim 1.6 \mathrm{~nm}$ for $1 \mathrm{~K}$ decrease in temperature. The surface stress at the top surface of the cantilever was found to be close to $0.25 \mathrm{MPa}$. This is significantly smaller than the capillary force resisting the release of the $\mathrm{ZnO}$ belt from the surface of the $\mathrm{Au}$ electrode. This significantly high force of adhesion keeps the $\mathrm{ZnO}$ belt in 
contact with the Au electrode for a large range of variation of temperature. However, decreasing the contact area (increasing the contact resistance) by decreasing the temperature results in a gradual decrease in the current. As the temperature is further decreased, owing to the inverse dependence of the Laplacian pressure on the distance, the adhesive force dramatically drops and the $\mathrm{ZnO} / \mathrm{PP}-\mathrm{BN}$ bimorph snaps off from the $\mathrm{Au}$ surface, resulting in an open circuit (OFF state of the switch in figure 3(c)). The switching temperature of the bimorph structure between and ON and OFF states can be precisely adjusted by controlling the thickness of the actuating polymer layer. Figure $3(\mathrm{~d})$ shows the plot of the temperature increase required to induce a deflection of $10 \mathrm{~nm}$ in the bimorph structure calculated using FEA. The arrow depicts the thickness of the plasma polymer layer $(100 \mathrm{~nm})$ discussed in this study. As can be seen from the plot, the temperature required to deflect the cantilever is highly sensitive to the thickness of the polymer layer. Assuming that the switch turns from $\mathrm{ON}$ to OFF state for a deflection of $10 \mathrm{~nm}$, the switching temperature can be tuned by changing the thickness of the polymer layer. It is also important to note that the capillary force and hence the ON/OFF transition temperature depends on the humidity of the ambient. The effect of humidity on the device performance is currently under investigation and will be reported in a separate publication.

\section{Conclusion}

In summary, we have demonstrated a thermal nanoswitch/ nanosensor based on a PP-BN/ZnO NB bimorph structure, which employs a novel read-out mechanism based on the variation of electric current with bimorph actuation. The bimorph demonstrated in this study obviates the use of complex microfabrication (to incorporate the piezoresistors into the cantilevers) or tedious optical alignment. We have demonstrated the unique combination of an electrical readout of thermal detection and a thermally triggered ON-OFF switch. Furthermore, the device architecture demonstrated here offers the ease of array fabrication for prospective thermal imaging or switching in an arrayed manner. The technology demonstrated here can also be adapted to realize a highly sensitive chemical and biological sensing system by altering the sensitive layer.

\section{References}

[1] Greene L E, Yuhas B D, Law M, Zitoun D and Yang P D 2006 Inorg. Chem. $457535-43$

[2] Wang Z L 2007 MRS Bull. 32 109-16

[3] Heo Y W, Norton D P, Tien L C, Kwon Y, Kang B S, Ren F, Pearton S J and LaRoche J R 2004 Mater. Sci. Eng. R $471-47$

[4] Huang M H, Mao S, Feick H, Yan H Q, Wu Y Y, Kind H, Weber E, Russo R and Yang P D 2001 Science 292 1897-9

[5] He J H, Ho S T, Wu T B, Chen L J and Wang Z L 2007 Chem. Phys. Lett. 435 119-22

[6] He J H and Ho C H 2007 Appl. Phys. Lett. 91233105
[7] Buchine B A, Hughes W L, Degertekin F L and Wang Z L 2006 Nano Lett. 6 1155-9

[8] He H, Hsin C L, Liu J, Chen L J and Wang Z L 2007 Adv. Mater. 19 781-4

[9] Law M, Kind H, Messer B, Kim F and Yang P D 2002 Angew. Chem. Int. Edn 41 2405-8

[10] Wang Z L and Song J H 2006 Science 312 242-6

[11] Qin Y, Wang X D and Wang Z L 2008 Nature 451 809-11

[12] Chang C Y et al 2006 Appl. Phys. Lett. 88173503

[13] Lee C Y, Haung Y T, Su W F and Lin C F 2006 Appl. Phys. Lett. 89231116

[14] Konenkamp R, Word R C and Godinez M 2005 Nano Lett. 5 2005-8

[15] Beek W J E, Wienk M M, Kemerink M, Yang X N and Janssen R A J 2005 J. Phys. Chem. B 109 9505-16

[16] Huynh W U, Dittmer J J and Alivisatos A P 2002 Science $2952425-7$

[17] Borghetti J, Derycke V, Lenfant S, Chenevier P, Filoramo A, Goffman M, Vuillaume D and Bourgoin J P 2006 Adv. Mater. 18 2535-40

[18] Gao Y H and Bando Y 2002 Nature 415599

[19] Dorozhkin P S, Tovstonog S V, Golberg D, Zhan J H, Ishikawa Y, Shiozawa M, Nakanishi H, Nakata K and Bando Y 2005 Small 1 1088-93

[20] Perazzo T, Mao M, Kwon O, Majumdar A, Varesi J B and Norton P 1999 Appl. Phys. Lett. 74 3567-9

[21] Jongeun C, Yamaguchi J, Morales S, Horowitz R, Zhao Y and Majumdar A 2003 Sensors Actuators A 104 132-42

[22] Senesac L R, Corbeil J L, Rajic S, Lavrik N V and Datskos P G 2003 Ultramicroscopy 97 451-8

[23] Zhao Y, Mao M Y, Horowitz R, Majumdar A, Varesi J, Norton P and Kitching J 2002 J. Microelectromech. Syst. 11 136-46

[24] Escriba C, Campo E, Esteve D and Fourniols J Y 2005 Sensors Actuators A 120 267-76

[25] LeMieux M C, McConney M E, Lin Y H, Singamaneni S, Jiang H, Bunning T J and Tsukruk V V 2006 Nano Lett. 6 $730-4$

[26] Lin Y H, McConney M E, LeMieux M C, Peleshanko S, Jiang C Y, Singamaneni S and Tsukruk V V 2006 Adv. Mater. 18 1157-61

[27] Wachter E A, Thundat T, Oden P I, Warmack R J, Datskos P G and Sharp S L 1996 Rev. Sci. Instrum. 67 3434-9

[28] Singamaneni S et al 2008 Adv. Mater. 20 1-29

[29] Datskos P G, Sepaniak M J, Tipple C A and Lavrik N 2001 Sensors Actuators B 76 393-402

[30] He J H, Lin Y H, McConney M E, Tsukruk V V, Wang Z L and Bao G 2007 J. Appl. Phys. 102084303

[31] Liu J, Gao P X, Mai W J, Lao C S and Wang Z L 2006 Appl. Phys. Lett. 89063125

[32] Haaland P and Targove J 1992 Appl. Phys. Lett. 61 34-6

[33] Tsukruk V V 1997 Rubber Chem. Technol. 70 430-67

[34] Ip K, Thaler G T, Yang H S, Han S Y, Li Y J, Norton D P, Pearton S J, Jang S W and Ren F 2006 J. Cryst. Growth 287149

[35] Kim S H, Kim H K and Seong T Y 2005 Appl. Phys. Lett. 86112101

[36] Sze S M 1981 Physics of Semiconductor Devices (New York: Wiley)

[37] Adamson A W 1997 Physical chemistry of Surfaces (New York: Wiley)

[38] Singamaneni S, LeMieux M C, Jiang H, Bunning T J and Tsukruk V V 2007 Chem. Mater. 19 129-31

[39] Chizhik S A, Huang Z, Gorbunov V V, Myshkin N K and Tsukruk V V 1998 Langmuir 14 2606-9 